\title{
Aplicación de inóculos líquidos de microorganismos nativos en el suelo: efecto en el crecimiento de plántulas de maíz
}

\author{
Application of native microbial inocula to the soil: effect on maize seedlings growth \\ Saúl Mendoza Sánchez ${ }^{1}$, Andrés Zepeda Hernández, \\ Erendira Gabriela Campeche Calixto ${ }^{1}$ y Fidel Adolfo Payán Zelaya ${ }^{1:}$
}

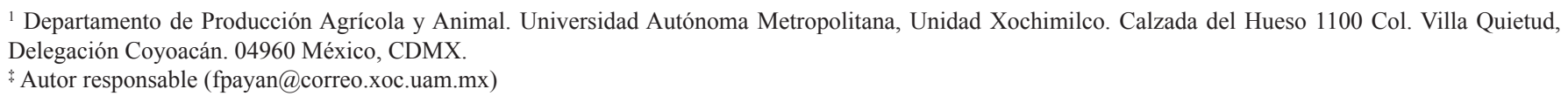

\section{RESUMEN}

La liberación de nutrientes provenientes de la descomposición de residuos vegetales en el suelo es importante para obtener una adecuada nutrición de cultivos sobretodo en sistemas agroforestales. Algunos autores han señalado la posibilidad de utilizar inóculos microbianos para acelerar tanto la descomposición de dichos residuos como el crecimiento de los cultivos. El objetivo de este trabajo fue evaluar la respuesta de plantas de maíz (Zea mays) a la aplicación de caldos nutritivos microbianos sobre residuos de Erytrhina americana en el suelo. Se condujo un experimento en condiciones semicontroladas en invernadero. El maíz se sembró en macetas llenas con suelo tratado con caldos nutritivos microbianos de seis diferentes localidades, los indicadores de la respuesta de las plantas de maíz fueron área foliar, biomasa y altura de la planta; se midió además producción de $\mathrm{CO}_{2}$ del suelo contenido en las macetas. Los resultados mostraron que área foliar, biomasa y producción de $\mathrm{CO}_{2}$ respondieron positivamente a la aplicación de follaje de Erytrhina americana. Sólo algunos caldos nutritivos microbianos afectaron significativamente las variables de respuesta evaluadas, y ninguno resultó superior a los resultados obtenidos con el tratamiento control que tenía suelo más residuos sin microorganismos. Los resultados señalan la necesidad de mayor investigación sobre la composición, dosis y frecuencia de aplicación de los inóculos y muestran la utilidad del método de comparación para encontrar diferencias en el impacto de los mismos.

Palabras clave: caldos microbianos, dióxido de carbono, Erythrina americana, follaje, suelo.

Cita recomendada:

Mendoza Sánchez, S. A. Zepeda Hernández, E. G. Campeche Calixto y F. A. Payán Zelaya. 2017. Aplicación de inóculos líquidos de microorganismos nativos en el suelo: efecto en el crecimiento de plántulas de maíz. Terra Latinoamericana 35: 161-168.

\section{SUMMARY}

The release of nutrients from decomposing plant residues is important to obtaining adequate crop nutrition especially in agroforestry systems. Some authors have pointed out the possibility of using microbial inocula to accelerate both residue decomposition and crop growth. In order to evaluate the response of maize (Zea mays) to application of liquid microbial inoculum on Erytrhina americana residues in the soil, an experiment was conducted in semi-controlled conditions. Maize was planted in pots filled with soil treated with microbial inocula from six different locations. Indicators of maize plant response were leaf area, biomass, and seedling height; $\mathrm{CO}_{2}$ production of soil in pots was also measured. The results showed that leaf area, biomass and $\mathrm{CO}_{2}$ production responded positively to the addition of foliage of E. americana. However, only microbial nutrient inocula from two locations caused significant differences in the variables evaluated, and none was superior to the results obtained with the control, which contained soil with residue addition but without microbial inoculants. The results indicate the need for further research on dose and frequency of application and they also highlight the usefulness of the method used to find differences in the impact of different inocula.

Index words: microbial broths, carbon dioxide, Erythrina americana, foliage, soil.

\section{INTRODUCCIÓN}

La sincronía entre la liberación de nutrientes provenientes de la descomposición de los residuos

Recibido: agosto de 2016. Aceptado: febrero de 2017. Publicado en Terra Latinoamericana 35: 161-168. 
arbóreos en el suelo y la demanda de nutrientes por los cultivos puede ayudar a obtener una adecuada nutrición de cultivos en sistemas agroforestales (Carrillo et al., 2011). Al utilizar Erythrina americana en suelos Typic Humitropept, se encontraron buenos resultados en el crecimiento de cultivos de maíz y mayores contenidos de $\mathrm{C}$ y $\mathrm{N}$ en suelos fertilizados con 9.2 Mg MS (materia seca) ha ${ }^{-1}$ año $^{-1}$ de residuos de poda (Bueno y Camargo, 2012; Martins et al., 2013). En experimentos bajo invernadero con plántulas de maíz, tasas más altas de crecimiento fueron observadas fertilizándolas con residuos de rápida descomposición provenientes de árboles fijadores de nitrógeno (Munguía et al., 2004).

El interés agronómico en la actividad microbiana se debe a su potencial para reciclar los nutrimentos, mejorar la nutrición de las plantas y disminuir o sustituir la aplicación de fertilizantes de origen industrial (Velasco et al., 2001; Alarcón et al., 2002).

Shintani y Tabora (2000) aceleraron la descomposición de residuos de banano utilizando una mezcla de inóculos microbianos. Velikonja et al. (2003) usaron otra mezcla microbiana para acelerar la descomposición de residuos vegetales urbanos con una ganancia de $28 \%$ en la mineralización del C en comparación con testigos no tratados. Se ha sugerido, utilizar las poblaciones microbianas como reservorios vivientes de nutrientes (Efthimiadou et al., 2010; Patel et al., 2010). En experimentos realizados con plántulas de maíz en macetas adicionadas con residuos de E. americana, Payán et al. (2013) obtuvieron mayores crecimientos utilizando inóculos microbianos con cepas nativas que sin ellas. Sin embargo, dichos experimentos originaron la necesidad de mayor investigación en cuanto a dosis y frecuencia de adición de los inóculos.

La búsqueda de estrategias para el manejo de las adiciones de biomasa arbórea utilizando la interacción entre poblaciones microbianas y la mesofauna con las condiciones del suelo, ha sido propuesta por Schroth (2003) para encontrar sistemas eficientes para reciclar los nutrientes a través de la descomposición de la materia orgánica. Teklay et al. (2007) plantearon que la sincronía entre la liberación de nutrientes provenientes de la descomposición de residuos y su demanda por parte de las plantas, puede mejorar la nutrición de cultivos en sistemas agroforestales. Por lo anterior, se plantea como problema científico la búsqueda de formas eficientes y económicamente viables para la manipulación de la materia orgánica del suelo, maximizando la toma de nutrientes pero manteniendo también los niveles de la misma a largo plazo, esto a través de la manipulación de poblaciones microbianas para la liberación y aprovechamiento de nutrientes provenientes de los residuos de poda de los árboles en sistemas agroforestales. Considerando esta problemática, los objetivos centrales de este trabajo fueron: analizar los efectos de mezclas microbianas de cepas nativas añadidas a los residuos de poda de E. americana y su incorporación al suelo, sobre el crecimiento de plántulas de maíz. Así como, estimar la producción de $\mathrm{CO}_{2}$ de los residuos de poda con y sin adición de mezclas microbianas y determinar la sensibilidad del método utilizado para detectar diferencias significativas entre distintas cepas microbianas obtenidas en diferentes localidades.

\section{MATERIALES Y MÉTODOS}

\section{Colecta de los Microorganismos}

La colecta de microorganismos se realizó en trampas de arroz colocadas al azar (diez frascos/sitio) durante siete días. En cada trampa, se colocó un frasco con $80 \mathrm{~g}$ de arroz, precocido y esterilizado. Una vez preparadas, se ubicaron en el suelo de tal manera que la boca del frasco quedara a ras de suelo, por último estas fueron cubiertas con hojarasca para conservar la humedad dentro de la trampa hasta el momento de ser retiradas. Las trampas fueron colocadas en cuatro zonas diferentes de la Ciudad de México donde se encontraban arboles de colorín (Erythrina poeppigiana): zoológico Los Coyotes, ubicado al sur de la ciudad en el ejido de San Francisco Culhuacán delegación Coyoacán; Centro de Investigaciones Biológicas y Acuícolas (CIBAC) a $300 \mathrm{~m}$ del embarcadero de Cuemanco; Centro de compostaje axolotl: Periférico Sur $\mathrm{s} / \mathrm{n}$ Parque Ecológico Xochimilco Delegación Xochimilco y en el bosque de Amecameca, al oriente del Estado de México.

\section{Preparación de Caldos Microbianos}

Se preparó la solución microbiana con una mezcla de $10 \mathrm{~L}$ de agua destilada, $189.17 \mathrm{~g}$ de soya texturizada molida, $208.33 \mathrm{~g}$ de yogurt, $33.33 \mathrm{~g}$ de arroz invadido por microorganismos nativos de las trampas, con una población mayoritariamente compuesta de hongos de 
los géneros Aspergillus, Trichoderma, Penicilium, determinados por observación de la morfología de las colonias que crecieron en las trampas, y $208.33 \mathrm{~g}$ de melaza. Se preparó el caldo microbiano con macerado de hojas deshidratadas de colorín que se molieron hasta obtener un polvo fino, se pesaron $150 \mathrm{~g}$ de las hojas en polvo y se vertieron en un bote con caldo microbiano, se almacenó a $4{ }^{\circ} \mathrm{C}$ hasta su uso. Para el control se preparó un caldo solo con las mismas cantidades de soya, yogurt y melaza sin inóculo.

\section{Experimento de Invernadero}

En el Centro de Investigaciones Biológicas y Acuícolas (CIBAC) de la Universidad Autónoma Metropolitana a $300 \mathrm{~m}$ del embarcadero de Cuemanco en el sur de la Ciudad de México, se implementó un experimento con un diseño completamente al azar con ocho tratamientos con cinco repeticiones cada uno, distribuyéndose de la siguiente manera: T0 (suelo testigo); T1 (suelo + hojas de colorín (HC); $\mathrm{T} 2$ (suelo $+\mathrm{HC}+$ caldo sin microbios); $\mathrm{T} 3$ (suelo + caldo con macerado $+\mathrm{HC}$ ); $\mathrm{T} 4$ (suelo $+\mathrm{HC}+$ caldo con microbios parque Coyotes); $\mathrm{T} 5$ (suelo $+\mathrm{HC}+$ caldo con microbios Amecameca); T6 (suelo + HC + caldo con microbios compostero CIBAC); T7 (suelo $+\mathrm{HC}+$ caldo con microbios Axolot). Cada unidad experimental se constituyó con una maceta de un litro de capacidad. Se cortaron hojas de colorín de una superficie de un $\mathrm{cm}^{2}$. El sustrato para las macetas fue $40 \mathrm{~kg}$ de suelo de la unidad experimental "Las Ánimas" en la zona de Tulyehualco, Ciudad de México; de una capa comprendida entre $10-20 \mathrm{~cm}$ de profundidad, se homogenizó con una tolva mezcladora, se dejó secar al sol durante una semana y por último se dejó en un lugar sombreado durante un mes, para estabilizar la actividad microbiana del suelo.

Para cada unidad experimental con tratamiento, se colocó un círculo de periódico en la base de la maceta, se vertió $1 \mathrm{~kg}$ de suelo y $26 \mathrm{~g}$ de cuadros de hoja de colorín, previamente homogenizado ( $8 \mathrm{~g}$ de materia seca), se asperjó una dilución microbiana (195 mL de agua destilada y $5 \mathrm{~mL}$ de caldo microbiano). Antes de usar el caldo microbiano se agitó vigorosamente. Se sembraron tres semillas de maíz por maceta, el suelo de todos los tratamientos fue mantenido a capacidad de campo con riegos de agua destilada cada tercer día. El experimento fue conducido bajo invernadero durante 60 días.
Cada tercer día se monitorearon las plantas, se registró la altura de la planta de mayor crecimiento. Al final del experimento se cortaron las plantas y se midió área foliar (AF), biomasa fresca (BF), biomasa seca (BS) de la planta mayor. La producción de $\mathrm{CO}_{2}$ emitida del suelo de la maceta como indicador de actividad microbiana, se midió con un analizador de gas infrarrojo PP SYSTEMS modelo EGM-4, la cámara de análisis se colocó sobre la superficie de cada maceta a las $48 \mathrm{~h}$ de haberse sembrado las plantas y antes de que hubiera germinación.

\section{Análisis Estadístico}

Los datos de las variables de respuesta fueron analizados para cada una de las fechas de medición, utilizando ANDEVA en un diseño completamente al azar de ocho tratamientos con cinco repeticiones. Los datos de respiración fueron analizados de la misma manera y cuando se detectaron diferencias significativas se aplicó la prueba Tukey de comparación de medias $P=0.05$.

\section{RESULTADOS Y DISCUSIÓN}

\section{Bioensayo con Plántulas de Maíz}

\section{Altura}

Los parámetros de crecimiento de las plantas de maíz fueron homogéneos desde la germinación, y a partir de los cinco días desde la siembra se observaron diferencias significativas $(P<0.05)$ entre los tratamientos. Los resultados obtenidos con el uso de hojas del colorín (E. americana) se tomaron en cinco puntos de medición (I, II, III, IV, V) de las 26 mediciones que se realizaron, tales puntos muestran mayor claridad la diferencia significativa que existía entre tratamientos (Figura 1).

En la medición realizada a partir del quinto día después de la emergencia de las plántulas de maíz (Punto I), únicamente el tratamiento T6 mostró un crecimiento significativamente menor a los demás que resultaron estadísticamente similares entre sí. A los 15 días (Punto II) después de la emergencia de las plántulas, T3 y T6 tuvieron una altura menor que los demás tratamientos. Estos resultados concuerdan con experimentos de invernadero donde se observó un mayor crecimiento de plantas de maíz como resultado 


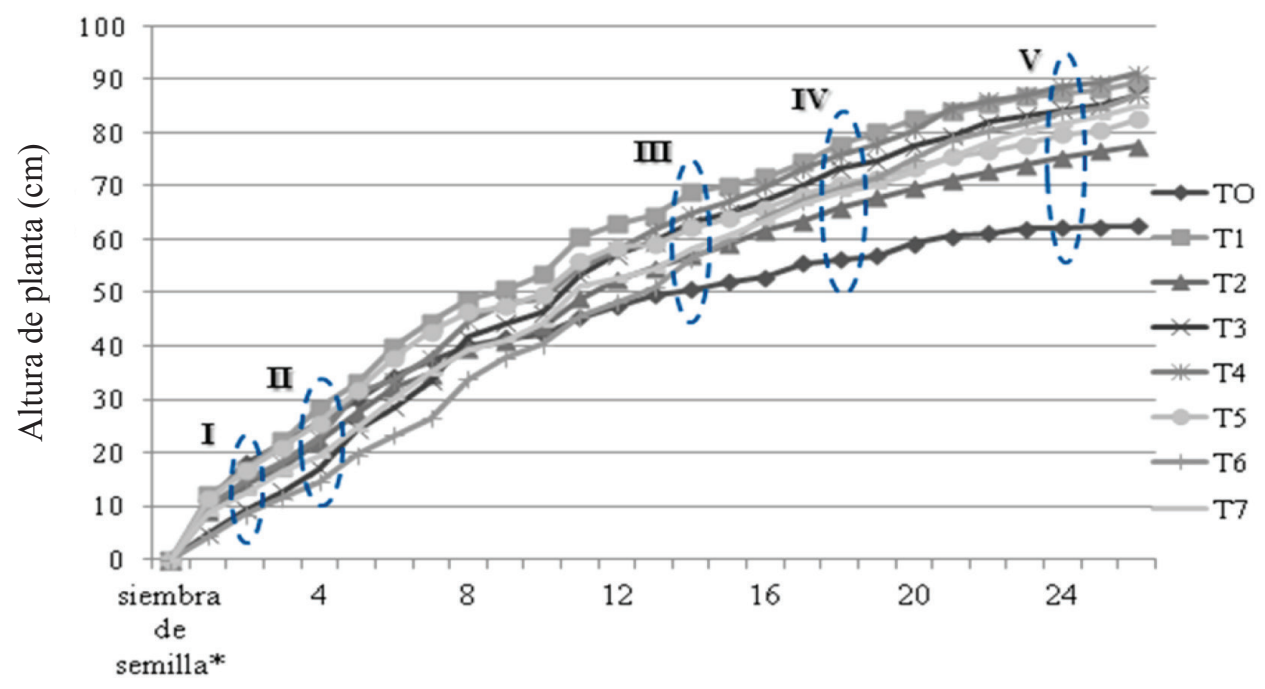

Mediciones

Figura 1. Altura de plantas de maíz de los diferentes tratamientos identificando los puntos (I, II, III, IV, V) que se tomaron para realizar pruebas estadísticas, ANDEVA y Tukey.

del arropado con residuos de poda del follaje de especies utilizadas como sombra en cafetales (Muñoz, 2002'; Munguía et al., 2004; Osorio 2004²). Y también se alinean con los obtenidos en un experimento con residuos de cuatro especies agroforestales etíopes Teklay et al. (2007), quienes propusieron formas de manejo de residuos con el mejoramiento de la actividad microbiológica. Sin embargo, las diferencias entre todos los tratamientos fueron observadas en un periodo muy corto, indicando que el flujo de nutrientes fue igualado sin adiciones artificiales de poblaciones microbianas durante los últimos días del experimento como resultado de un proceso de homeostasis, en el cual las poblaciones microbianas tienden a alcanzar un equilibrio entre ellas tanto en su número, como en el aprovechamiento de los nutrientes disponibles, regresando al estado inicial del experimento, tal como lo plantea Alvarenga et al. (2013). Por otra parte no se observaron diferencias en crecimiento en ese periodo entre las plántulas abonadas solo con hojas (T1) y las que fueron inoculadas, señalando la falta de efectividad de dicha inoculación en ese periodo experimental. En el experimento reportado se observaron morfológicamente los géneros Aspergillus, Trichoderma, Penicilium, que son hongos del suelo con actividad degradadora de materiales lignocelulócicos. En un experimento similar Payán et al. (2013) detectaron los géneros Aspergillus, Trichoderma, Verticilium, Metarizzum y Gliocadium así como 6 cepas de bacterias de tinción gram+, que no pudieron ser identificados. De acuerdo a Fischersworring (2001) se puede esperar un incremento en la descomposición por la acción del consorcio microbiano total sin necesidad de una identificación mas específica. Más aún, en algunos casos en la medición a los 15 días de la emergencia de las plántulas el tratamiento sin inoculo tuvo crecimiento significativamente mayor que los tratamientos T3 y T6 que si fueron inoculados.

En la medición de 38, 49 y 63 días (Puntos III, IV y V) después de la emergencia, solamente el testigo T0 fue significativamente menor que todos los demás, este efecto de los inóculos microbianos se le atribuye a los residuos de E. americana que produjeron una alta actividad microbiana, medida indirectamente a través de la producción de $\mathrm{CO}_{2}$, en consecuencia de la incorporación de materiales de fácil descomposición que no provocan una acumulación "envejecida" de materia orgánica. De acuerdo a Munguía et al. (2004) la disponibilidad de $\mathrm{N}$ en parcelas con cobertura vegetal de E. americana fue mayor que el suelo desnudo debido a la acumulación a largo plazo de la MOS y N orgánico, concluyendo así que los residuos añadidos recientemente contribuyen sólo en una pequeña proporción al $\mathrm{N}$ mineral absorbido por las plántulas de maíz, basándose en las altas tasas de descomposición de los residuos de E. americana (Figura 2).

\footnotetext{
${ }^{1}$ Muñoz, C. Y. 2002. Disponibilidad de nutrimentos de tres compostas. MScThesis, CATIE, Turrialba, Costa Rica 66 pp.

${ }^{2}$ Osorio M.,V. E. 2004. Descomposición y liberación de nitrógeno de material foliar y radicular de siete especies de sombras en un sistema agroforestal con café. MScThesis, CATIE, Turrialba, Costa Rica 75 pp.
} 


\section{Área Foliar y Biomasa}

Al evaluar el área foliar a los 63 días después de la germinación de las semillas, se encontró que las plantas cultivadas en las macetas inoculadas con caldos nutritivos microbianos de colorín (T4) y de estiércol CIBAC (T6), mostraron un incremento en la magnitud de dicha variable, respecto a los tratamientos T2, T3, y T7, incluyendo el tratamiento control T0 y fueron estadísticamente iguales a los tratamientos T1 y T5 (Figura 3). Las diferencias significativas $(P<0.05)$ en alturas de planta, solo se observaron entre algunos tratamientos microbianos 15 días después de la emergencia, y el tratamiento sin microbios no se diferenció de los tratados, lo que indica que si los nutrientes de los residuos de poda se conservaron en la biomasa microbiana, no se dio una mayor liberación de nutrientes por lo menos en los 63 días de duración
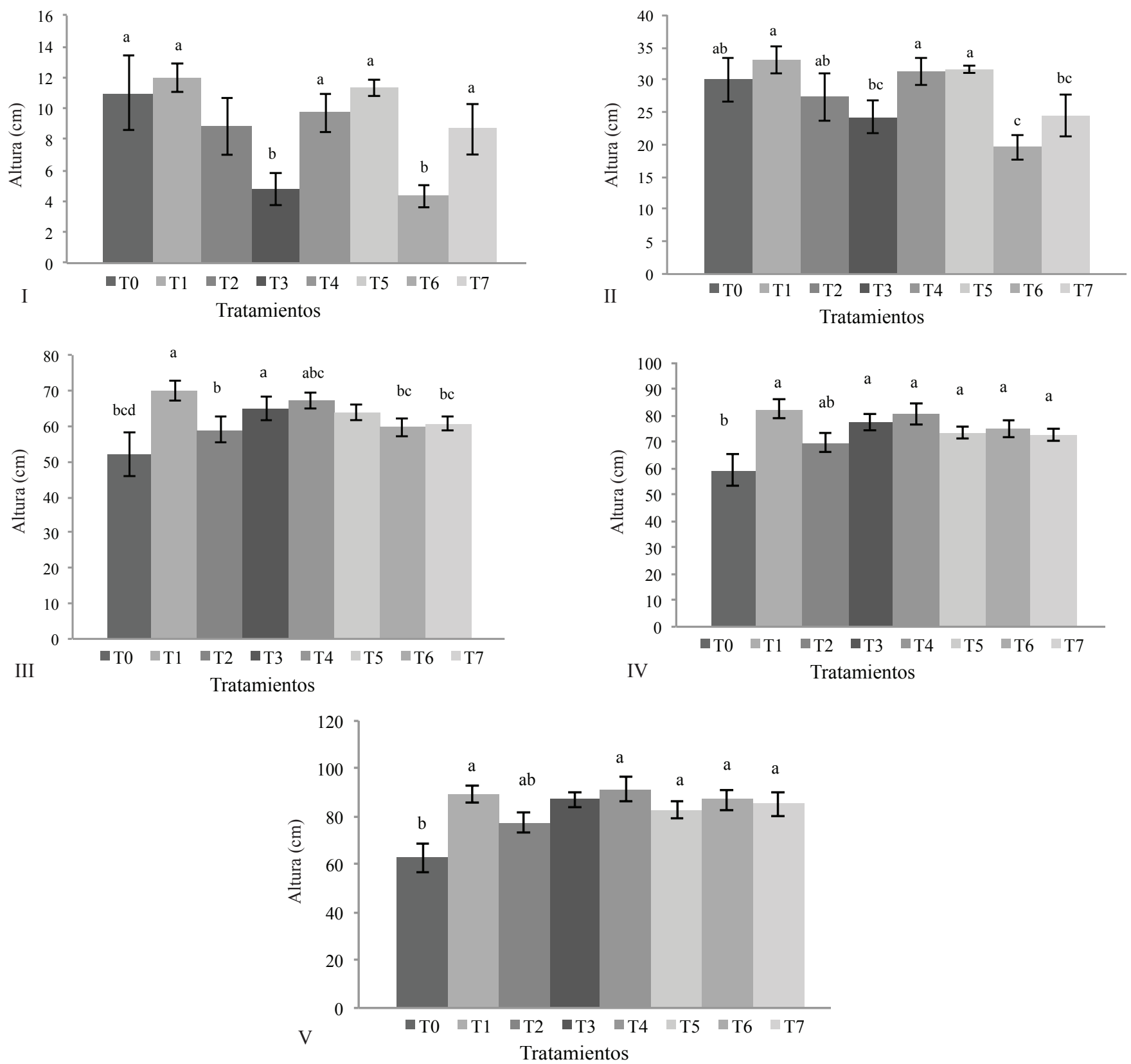

Figura 2. Altura de plantas de maíz de los diferentes tratamientos en los puntos I, II, III, IV, V. Columnas con letras iguales no son significativamente diferentes. 


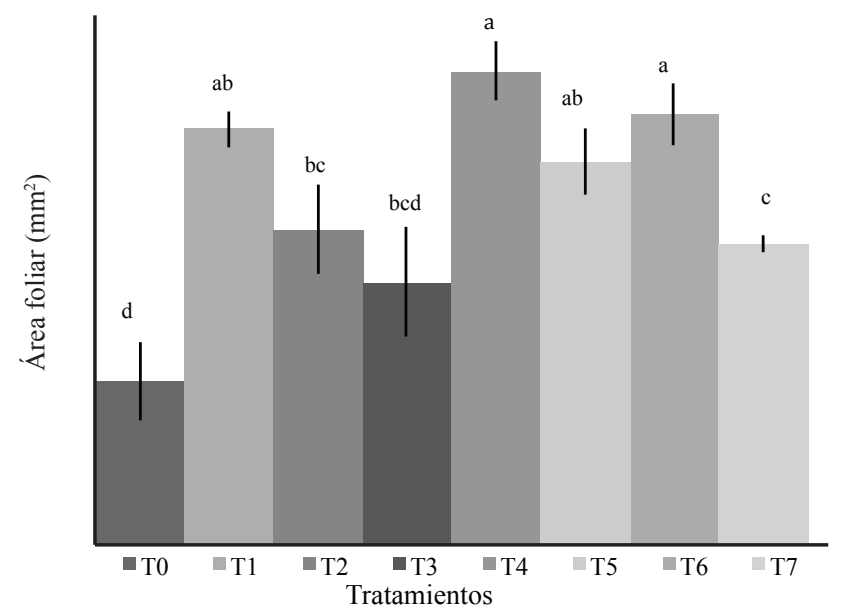

Figura 3. Área foliar de plántulas de maíz con siete tratamientos microbianos después de $\mathbf{6 3}$ días de la primera aplicación de inóculos. Valores con las mismas letras no son significativamente diferentes.

del experimento. Este resultado pudo deberse a la falta de un inoculo adecuado o a la falta de actividad del mismo ya que Patel et al. (2010) encontraron que la productividad de arroz (Oryza sativa) en la India se mejoró debido a un aumento en biomasa microbiana del suelo concomitantemente con una mayor liberación de nutrientes 50 días después de la aplicación de un abono verde, manteniendo un flujo anual de $\mathrm{N}$ a través de la biomasa microbiana de 30-45 $\mathrm{kg} \mathrm{ha}^{-1}$ año $^{-1}$.

La biomasa (peso seco de follaje, tallo y raíces) al final del experimento solo mostró diferencias significativas $(P<0.05)$ entre los tratamientos y el control T0, y fueron estadísticamente similares entre ellos (Figura 4).

\section{Producción de $\mathrm{CO}_{2}$ del Suelo}

Las mediciones de $\mathrm{CO}_{2}$ y la segunda y tercera medición de la altura junto con la medición final de área foliar fueron las variables más sensibles a las diferencias entre tratamientos. Particularmente la de $\mathrm{CO}_{2}$ antes de la emergencia permitió ver que aquellos tratamientos con mayor actividad microbiana(reflejada por las emisiones) mostraron menores tasas de crecimiento. (T3 Figura 2 y Figura 5). Por el contrario los tratamientos sin aplicaciones microbianas y solo adición de hojas (T1 Figura 2 y Figura 5) mostraron tendencia a tasas superiores o al menos similares a las que si tenían aplicaciones. Lo cual apoyaría

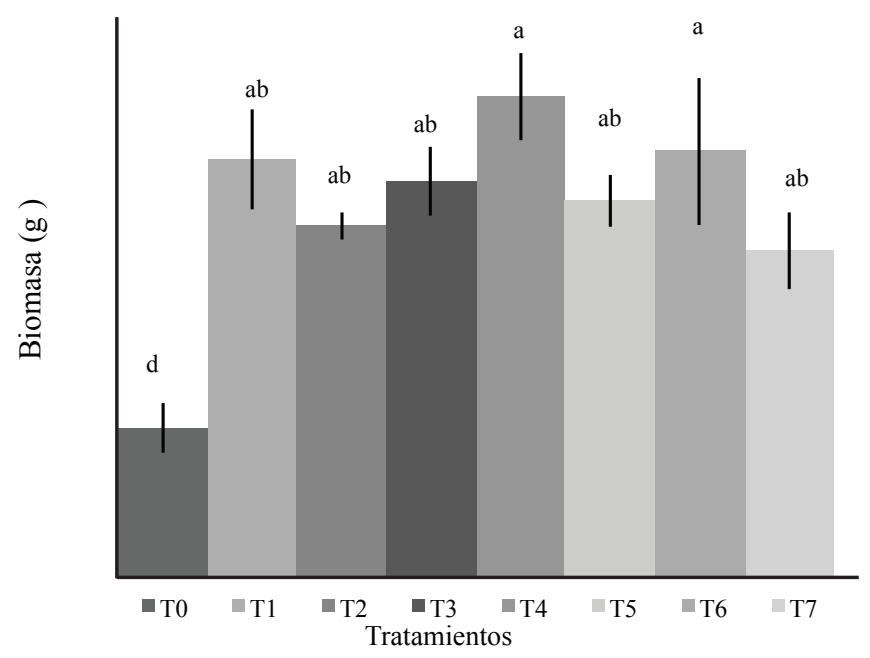

Figura 4. Producción de biomasa. Letras iguales corresponden a valores que no son significativamente diferentes. 


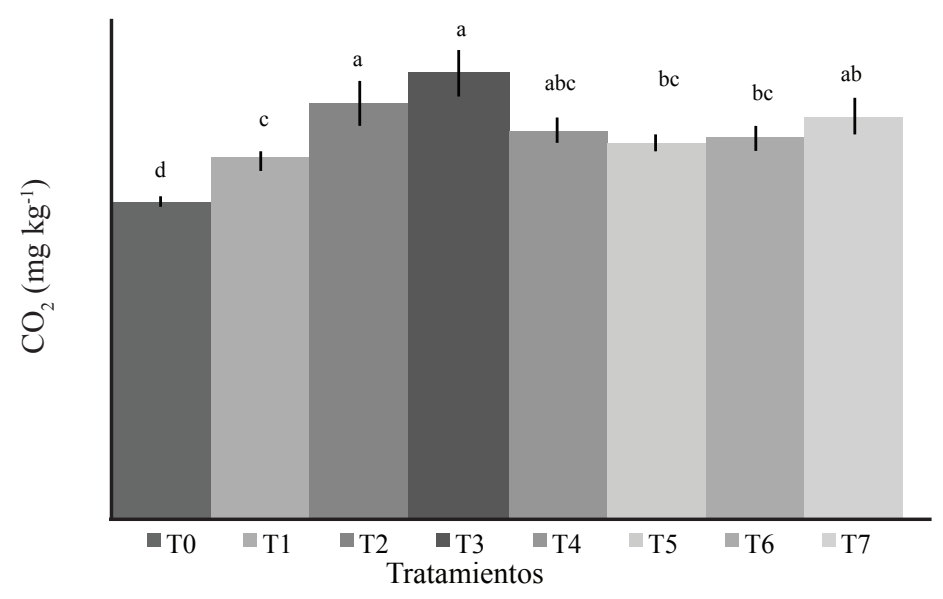

Figura 5. Producción de $\mathbf{C O}_{2}$. Letras iguales indican que no hay diferencias significativas entre los valores correspondientes.

la hipótesis de que ocurrió una inmovilización de nutrientes al menos en las primeras dos semanas del experimento que impidió un mayor crecimiento de plántulas. La sensibilidad de la medición de $\mathrm{CO}_{2}$ a la adición de inóculos microbianos es congruente con lo observado por Payán et al. (2013), en un experimento en que se midió el $\mathrm{CO}_{2}$ después de la aspersión de microorganismos descomponedores a fracciones aisladas de la materia orgánica del suelo.

Por otra parte a los tres días de sembradas las semillas y previo a la emergencia de las plántulas los tratamientos T3 y T2 mostraron una producción de $\mathrm{CO}_{2}$ superior a los tratamientos $\mathrm{T} 6 \mathrm{y} \mathrm{T} 1 \mathrm{y}$ al testigo, todos los demás tratamientos no mostraron diferencias (Figura 5). Es de notar que el tratamiento de mayor respiración fue el que se detectó como el de menor crecimiento en el punto II de la medición de altura. A pesar de que mayor respiración, indicaría que hay mayor descomposición de la MO y disponibilidad de nutrientes para la planta, entonces indirectamente debió favorecerse el mayor crecimiento de la planta . Sin embargo los datos indican que los nutrientes fueron tal vez inmovilizados en la biomasa microbiana o perdidos por volatilización. La sincronía entre la mayor actividad microbiana expresada en producción de $\mathrm{CO}_{2}$ con los mayores flujos naturales de liberación de nutrientes que ocurren en residuos de fácil degradación como los de colorín pudieron haber enmascarado la actividad de las mezclas microbianas, pues pueden haber sido llevadas a cabo muy cerca del flujo natural de liberación de nutrientes. Por otro lado podrían utilizarse residuos más resistentes a la descomposición para obtener resultados más efectivos.

Las mediciones realizadas también proveen una indicación sensitiva de la respuesta de la actividad microbiana a variaciones de temperatura y humedad, los efectos de humedecimiento-secado, la aplicación de agroquímicos o elementos metálicos, la exudación de sustancias supresoras y el manejo del medio, o como en nuestro caso la adición de materiales ricos en C y N (García et al., 2003; Peña, 2004³).

\section{CONCLUSIONES}

- La falta de incrementos significativos en el crecimiento de las plántulas y diferencias significativas entre los tratamientos con microbios de cuatro distintas localidades, restringido temporalmente a las primeras dos semanas del experimento sugiere un limitado efecto de la adición de las soluciones microbianas aceleradoras de la descomposición de los residuos de poda utilizadas en este experimento.

- Una recomendación de su uso por tanto resultaría injustificada y menos en suelos con fertilidad media. - La igualación de las tasas de crecimiento después de 17 días también indica que no se dio una mayor disponibilidad de los nutrientes provenientes de los residuos a través del uso de las soluciones microbianas nativas o se requiere de mayor tiempo para alcanzarla. - Debido a utilización de residuos de muy fácil descomposición como los de E. americana,

${ }^{3}$ Peña, W. 2004. Los suelos desarrollados sobre serpentinitas y su relación con la flora endémica. Índice bioquímico y metales. Tesis Doctoral, Universidad de Santiago de Compostela y CSIC, España. 404p. 
la velocidad del proceso no puede ser acelerado por lo que sería recomendable realizar estudios con materiales arbóreos de lenta descomposición.

- Se propone la realización de nuevos estudios sobre dosis, calidad del inoculo y espaciamiento entre las aplicaciones de inóculos microbianos.

\section{LITERATURA CITADA}

Alarcón, A., F. T. Davies, J. N. Egilla, T. C. Fox, A. A. EstradaLuna, and R. Ferrera-Cerrato. 2002. Short term effects of Glomusclaroideum and Azospirillum brasilense on growth and root acid phosphatase activity of Carica papaya L. under phosphorus stress. Rev. Latinoam. Microbiol. 44: 31-37.

Alvarenga, P., C. Laneiro, P. Palma, A. de Varennes, and A. C. Cunha-Queda. 2013. A study on $\mathrm{As}, \mathrm{Cu}, \mathrm{Pb}$ and $\mathrm{Zn}$ (bio) availability in an abandoned mine area (Sao Domingos, Portugal) using chemical and ecotoxicological tools. Environ. Sci. Pollut. Res. 20: 6539-6550.

Bueno, L. y J. C. Camargo. 2012. Cambios del nitrógeno durante etapas de desarrollo temprano de la leucaena (Leucaenaleucocephala (Lam) de Wit). Liv. Res. Rural Dev. 24, http://www.lrrd.org/lrrd24/2/buen24033.htm

Carrillo, Y., C. F. Jordan, K. L. Jacobsen, K. G. Mitchell, and P. Raber. 2011. Shoot pruning of a hedgerow perennial legume alters the availability and temporal dynamics of root-derived nitrogen in a subtropical setting. Plant Soil 345: 59-68.

Efthimiadou, E., E. M. Papatheodorou, N. Monokrousos, and G. P. Stamou. 2010. Changes of soil chemical, microbiological, and enzymatic variables in relation to management regime and the duration of organic farming in Phaseolus vulgaris. J. Biol. Res. Thessalon. 14: 151-159.

Fischersworring, B. y R. Roßkamp. 2001. Guía para la caficultura ecológica. GTZ. Popayán, Colombia.

García, C., F. Gil-Sotres, T. Hernández y C. Trasar-Cepeda. 2003. Técnicas de análisis de parámetros bioquímicos en suelos: Medida de actividades enzimáticas y biomasa microbiana. Mundi Prensa. Madrid.
Martins, S. C., J. Galmés, A. Molins, and F. M. DaMatta. 2013. Improving the estimation of mesophyll conductance to $\mathrm{CO}_{2}$ : On the role of electron transport rate correction and respiration. J. Exp. Bot. 64: 3285-3298.

Munguía, R., J. Beer, J. M. Harmand y J. Haggar. 2004. Tasas de descomposición y liberación de nutrientes de la hojarasca de Eucalyptus deglupta, Coffea arabica y de hojas verdes de Erythrina poeppigiana solas y en mezclas. Agroforestería en las Am. 11: 62-68.

Patel, K., J. I. N. Kumar, R. N. Kumar, and R. K. Bhoi. 2010. Seasonal and temporal variation in soil microbial biomass $\mathrm{C}$, $\mathrm{N}$ and $\mathrm{P}$ in different types land uses of dry deciduous forest ecosystem of Udaipur, Rajasthan, Western India. Appl. Ecol. Environ. Res. 8: 377-390.

Payán-Zelaya, F, J. M. Harmand, A. Flores-Macías, J. Beer, G. Ramos-Espinoza, and F. De Léon González. 2013. Soil nutrient availability and $\mathrm{C}_{2}$ production in agroforesty systems after de addition of Erythrina poeppigiana pruning residues and native microbial inocula. Agrofor. Syst. 87: 439-450.

Schroth, G. 2003. Decomposition and nutrient supply from biomass. pp. 131-150. In: G. Schroth and F. L. Sinclair (eds.). Trees, crops and soil fertility concepts and research methods. CABI Publishing, Bristol,

Shintani, M. and P. Tabora.2000. Organic fertilizer: Managing banana residues with effective microorganism (EM). In: T. Alfoldi, W. Lockeretz, and U. Niggli (eds.). The world grows organic. Proceedings 13th International IFOAM Scientific Conference. Zurich, Switzerland. ISBN: 3-7281-2754-X.

Teklay, T., A. Nordgren, G. Nyberg, and A. Malmer. 2007. Carbon mineralization of leaves from four Ethiopian agroforestry species under laboratory and field conditions. Appl. Soil Ecol. 35: 193-202.

Velasco V., J., R. Ferrera-Cerrato y J. J. Almaráz-Suárez. 2001. Vermicomposta, micorriza arbuscular y Azospirillum brasiliense en tomate de cáscara. Terra 19: 241-248.

Velikonja, S., R. Mihelic, F. Lobnik, and D. Lestan. 2003. Microbial community structure during composting with and without mass inocula. Comp. Sci. Util. 11: 6-15. 